\title{
MYOCARDIAL REVASCULARIZATION AFTER ACUTE MYOCARDIAL INFARCTION BY THROMBOSED CORONARY ANEURYSM IN BEHÇET'S SYNDROME
}

Paula de Medeiros Nacácio e Silva ${ }^{1, \star}$, Renata Maria Monteiro Pinto ${ }^{1}$, Marina de Azevedo Martins ${ }^{1}$, Danielle Arraes Rubini ${ }^{1}$, Stan Richard Medeiros de Souza ${ }^{1}$, Lucas Victória de Oliveira Martins ${ }^{1}$, Renata Ferreira Rosa1, Rina Dalva Neubarth Giorgi ${ }^{1}$, Nafice Costa Araújo ${ }^{1}$

1.Hospital do Servidor Público Estadual, São Paulo (SP), Brazil.

*Corresponding author: paula.nacacio@gmail.com

\section{BACKGROUND}

Behçet's syndrome (BS) is a vasculitis that must be considered in the context of recurrent oral and genital ulcers, accompanied by systemic manifestations, such as uveitis, arthritis, skin lesions, neurological disease or vascular disease. It can involve blood vessels of all sizes (small, medium and large), affecting both arterial and venous circulation. Rarely, BS can involve coronary arteries with ischemic complications.

\section{CASE REPORT}

A previously healthy 18-year-old male presented with sudden acute chest pain being diagnosed with non-ST segment elevation acute myocardial infarction. The patient underwent coronary cineangiography which demonstrated a thrombosed saccular aneurysm in proximal anterior descending coronary artery. Patient reported a history of recurrent and painful oral and genital ulcers and acneiform skin rash. On physical examination, the patient had skin lesions in venipuncture sites that resembled pathergy and eye fundoscopy showed retinal artery aneurysm. Laboratory tests revealed elevated erythrocyte sedimentation rate $(80 \mathrm{~mm} / \mathrm{h})$. A diagnosis of Behçet's disease with important vascular involvement was made. Initially, the patient was treated with oral corticosteroids (prednisone $1 \mathrm{mg} / \mathrm{kg} /$ day) and intravenous cyclophosphamide $\left(0.5 \mathrm{~g} / \mathrm{m}^{2}\right)$, then underwent myocardial revascularization with mammary graft for anterior descending artery. The postoperative period occurred without complications. Follow-up after 3 months, the patient is well, tapering prednisone, without complaints of chest pain and has complete resolution of mucocutaneus lesions and normal erythrocyte sedimentation rate.

\section{CONCLUSION}

Behçet's syndrome is a variable vessels vasculitis that can affect virtually any blood vessels. The involvement of coronary vessels in BS is relatively rare without estimated prevalence, and can manifest as coronary arteritis or coronary aneurysm. The coronary aneurysm can be calcified or saccular and mural thrombosis can be seen. Also, coronary aneurysm can occur isolated or in association with others aneurysms, such as retinal aneurysm in this case. Clinically, it can manifest as angina or acute myocardial infarction. Due to the risk of rupture, the coronary aneurysm should be treated with revascularization surgery. As proposed, if possible, severe vascular involvement in BS, such as coronary aneurysm, should be treated with high dose corticosteroids (prednisone $1 \mathrm{mg} / \mathrm{kg} / \mathrm{day}$ or intravenous methylprednisolone) plus cyclophosphamide or TNF inhibitors before the surgical procedure to reduce the risk of post-surgical complications due to the vasculitis. This case report shows a rare involvement of BS which immunosuppressive and surgical treatment were associated with a good clinical outcome. 